\title{
Caracterização da produção dos docentes/pesquisadores dos cursos de educação física da Paraíba
}

Lívia Tenório Brasileiro

Professora Adjunta da Universidade de Pernambuco

Roseane Barros da Silva

Graduada em Educação Física pela UEPB

Dianderson Carlos Costa Silva

Graduando em Educação Física na UEPB

\section{Resumo}

Esta pesquisa visa contribuir com a análise da trajetória e das tendências das pesquisas desenvolvidas na região Nordeste, apresentando como objetivos: identificar as produções dos docentes/pesquisadores (mestres e doutores) que atuam na região Nordeste do Brasil, com ênfase no Estado da Paraíba; identificar as tendências temáticas e teórico-metodológicas; identificar as contribuições dessa produção na formação profissional e na consolidação da pesquisa nas instituições formadoras da região.

Palavras-Chave: Produção de Conhecimento; Educação Física; Paraíba.

\begin{abstract}
This research aims to contribute to the analysis of the trajectory and tendencies of research developed in the Northeast, with the following objectives: identify the productions of teachers/ researchers working in Northeastern Brazil, with emphasis on the state of Paraíba; identify tendencies thematic and theoretical and methodological; identifying the contributions of this production in vocational training and consolidation of research in educational institutions in the region.
\end{abstract}

Keywords: Production of knowledge; Physical Education; Paraíba. 


\section{Introdução}

presente estudo é parte constitutiva do projeto temático:
Produção científica em Educação Física no Nordeste do Brasil:
os impactos do sistema de pós-graduação - região Sudeste - na produção de docentes, mestres e doutores e na implementação da pesquisa nas instituições formadoras da região Nordeste (CHAVES-GAMBOA; SÁNCHEZ GAMBOA; TAFFAREL, 2009).

Toma como referência a pesquisa de Chaves (2005), intitulada Epistemologia da Educação Física - EPISTEF: a produção científica na Educação Física nos estados do Nordeste, desenvolvida no grupo LEPEL/FACED/UFBA, que mapeou e analisou as produções docentes das instituições formadoras dos estados de Alagoas, Bahia, Pernambuco e Sergipe, entre os anos de 1982 a 2004. Neste momento, o estudo pretende atualizar e ampliar o mapeamento e análise da produção dos docentes de Educação Física dos nove estados da região Nordeste, incluindo nesta etapa o Ceará, o Maranhão, a Paraíba, o Piauí e o Rio Grande do Norte.

O projeto vem sendo desenvolvido desde 2010 e ampliou sua discussão junto às equipes estaduais da região Nordeste em 2011, e em 2012 recebeu financiamento FAPESP - proc. 2012/50019-7. Desde 2010 a equipe da Paraíba se inseriu no estudo, tendo recebido bolsa de Iniciação Científica PIBIC/UEPB/CNPq entre 2010/2011 - 2011/2012 e 2012/2103.

Tal pesquisa visa contribuir com a análise da trajetória e das tendências das pesquisas desenvolvidas na região, identificando áreas temáticas, opções teórico-metodológicas e caracterização das correntes e perspectivas das mesmas, assim como desdobramentos e contribuições para a formação profissional da área.

Apresentando como objetivos: identificar as produções dos docentes/pesquisadores (mestres e doutores) que atuam na região Nordeste do Brasil, com ênfase no Estado da Paraíba; identificar as tendências temáticas e teórico-metodológicas; identificar as contribuições dessa produção na formação profissional e na consolidação da pesquisa nas instituições formadoras da região. 


\section{Dossiê Análise da produção do conhecimento}

Os dados ora apresentados são resultados da segunda etapa da pesquisa, entre os anos de 2011/2012, sistematizados nos relatórios de pesquisa PIBIC/UEPB/CNPq (BRASILEIRO; SILVA, 2011, BRASILEIRO; SILVA, 2012.

\section{O cenário da formação em educação física na Paraíba}

A partir do acesso ao sistema do Ministério de Educação - e-MEC, identificamos mais de 480 Instituições do Ensino Superior - IES existentes nos 09 estados da região Nordeste, destas cerca de 90 IES oferecem cursos de Educação Física, em suas duas possibilidades de modalidade: Licenciatura e Bacharelado, havendo a maior concentração no estado da Bahia e a menor no estado de Sergipe.

$\mathrm{Na}$ Região Nordeste o primeiro registro de curso de Educação Física é na Universidade de Pernambuco criado em 1946. Porém, será na década de 1970 que as universidades federais ampliaram a ocorrência de cursos: em 1973 iniciam os cursos da Universidade Federal do Rio Grande do Norte UFRN e da Universidade Federal de Pernambuco - UFPE; em 1974 nasce o curso da Universidade Federal de Alagoas - UFAL; em 1975 é criado o curso da Universidade Federal de Sergipe - UFS; em 1976 instala-se o curso da Paraíba na Universidade Federal da Paraíba - UFPB; em 1977 inicia o curso da Universidade Federal do Maranhão - UFMA; em 1978 o curso da Universidade Federal do Piauí - UFPI; e só em 1988 o curso da Universidade Federal da Bahia - UFBA. Entrando na década de 90 com o curso da Universidade Federal do Ceará - UCE em 1993. Outras instituições estaduais e privadas são criadas também na década de 1970, entre elas: em 1972 a Universidade do Ipê, hoje Centro Universitário de João Pessoa UNIPÊ, primeiro curso da Paraíba; em 1973 nascem os cursos da Universidade de Fortaleza - UNIFOR e da Universidade do Vale do Acaraú - UVA, primeiros no Ceará, e mais um na Universidade do Estadual do Rio Grande do Norte - UERN, e na Universidade Católica de Salvador UCSAL; em 1979 é criado o curso da Universidade Estadual da Paraíba UEPB.

Reconhecemos que a ampliação dos cursos de Educação Física em 
nossa região acompanha o cenário nacional, que tem a década de 1970 como sendo o período de maior ampliação dos cursos de formação, especialmente os de Licenciatura, curta e plena, para dar conta da demanda anunciada na legislação brasileira para atender a escolarização básica.

O Estado da Paraíba, nosso campo de estudo, possui 36 Instituições de Ensino Superior, sendo 03 universidades públicas: 01 Estadual (UEPB) e 02 Federais (UFPB - Universidade Federal de Campina Grande - UFCG), além de 01 Centro Federal de Educação Tecnológica (CEFET-PB); e 32 Instituições Privadas.

Dentre essas instituições, são oferecidos, em todo o Estado, 08 Cursos de Educação Física, sendo 04 em instituições públicas (UEPB - UFPB Universidade de Brasília - UNB) e 04 em instituições privadas (UNIPÊ; Faculdades Integradas de Patos - FIP; Faculdade Mauricio de Nassau FMN/JP), sendo o curso da UNB à distância.

A UNIPE instalou o primeiro Curso de Licenciatura em Educação Física na Paraíba, iniciando suas atividades em 13/03/1972 e com autorização do MEC em 27/12/1976. Em seguida a UFPB tem curso Licenciatura em Educação Física, através de Resolução CONSUN/UFPB em 24/03/1977, iniciando suas atividades em 18/08/1976 e com autorização do MEC em 31/10/1978. E a UEPB tem curso de Licenciatura em Educação Física através de Resolução CONSEPE/UEPB em 26/05/1978, iniciando suas atividades em 05/03/1979 e com autorização do MEC em 25/10/1984.

\section{$\mathrm{O}$ acesso às produções}

O estudo está baseado em uma pesquisa documental. Para localizar as produções dos docentes/pesquisadores acompanhamos as seguintes etapas:

- Identificação, nas páginas virtuais dos cursos de Educação Física das

IES da Paraíba, a composição do corpo docente, selecionando os mestres e doutores que atuam nos mesmos. Em alguns casos foi necessário o contato direto com os coordenadores de cursos, visto que o corpo docente não estava disponível on line;

- Acesso ao Currículo Lattes/Conselho Nacional de Pesquisa - CNPq dos docentes identificados, de forma a localizar os títulos, anos e programas de pós-graduação de suas produções de mestrado e doutorado; 
- Localização das produções, oriundas de programas brasileiros, no Banco de Teses da Coordenação de Aperfeiçoamento de Pessoal de Nível Superior - CAPES, que disponibilizou os seguintes dados: título, ano, orientador(es), programa, área, linha, banca examinadora, palavras-chave, fomento, biblioteca depositaria e resumo;

- Localização dos textos completos através das bibliotecas virtuais das instituições dos programas de pós-graduação e outros repositórios disponíveis.

Nesta etapa do projeto centramos nossa ação na ampliação das informações do primeiro grupo de IES públicas do Estado: UEPB e UFPB e finalizamos a catalogação das IES privadas: FIP, FMN/JP, além de inserirmos o curso a distância da UNB, ficando uma instituição - UNIPÊ de fora da pesquisa, por não ter disponibilizado os dados do corpo docente, mesmo que solicitado reiteradamente no ano de 2012.

Os dados reunidos nos permitiu atualizar e preencher as seguintes planilhas, em excel:

- Planilha I - Cursos de Educação Física em atividade nos Estados da Paraíba;

- Planilha II - Levantamento dos nomes dos docentes (mestres e doutores) que atuam nos cursos de Educação Física no Estado da Paraíba;

- Planilha III - Instrumento de registro da caracterização da produção científica.

Os dados das produções disponíveis foram lidos e analisadas, procurando identificar os tópicos da fase 1 da planilha III: código de registro; estado; sigla da IES; código da IES; nome do docente; gênero do docente; link currículo lattes; área de formação da graduação; titulação (mestrado e doutorado); IES da titulação; área de pós-graduação; linha e/ou grupo de pesquisa; agência de fomento; título da pesquisa; localização da pesquisa; ano de defesa; orientador; gênero do orientador; resumo; palavraschave. 


\section{Caracterização das produções}

Recorreu-se, nesta etapa, às Informações referentes aos docentes/pesquisadores que atuam nos 08 cursos de Educação Física registrados nas 05 IES da Paraíba (UFPB, UEPB, UNIPÊ, FIP e FMN/JP) e 01 instituição de ensino a distância (UNB), sendo identificadas as pesquisas da pós-graduação stricto sensu por eles produzidas.

Identificou-se 90 IES que possuem cursos de Educação Física nos 09 estados do Nordeste, sendo 05 (5,68\%) na Paraíba, somada a 01 da região Centro-Oeste com um curso à distância. Destas 03 são públicas (UFPB, UEPB, UNB), 03 privadas (UNIPÊ, FMN/JP, FIP), que totalizam 08 ocorrências de cursos, sendo 04 licenciaturas e 04 bacharelados.

Para nossa análise totalizamos 16 docentes na UEPB, 23 na UFPB, 20 na UNB, 15 na FMN/JP, 10 na FIP. Destes 36 são mulheres e 48 são homens, havendo uma pequena disparidade de gênero nesta composição.

Dos 84 docentes há uma concentração com formação inicial em Educação Física, totalizando: 82 formados em Educação Física e 02 em Fisioterapia, havendo o registro de outras formações para além da Educação Física em alguns casos, com cursos de Teologia, Jornalismo, Psicologia e Filosofia.

Estes 84 docentes registram 131 produções, tendo maior concentração na UFPB, instituição local e UNB instituição externa, e os menores índices nas instituições privadas, conforme quadro 1 e 2 .

Quadro 1 - Número de docentes dos Cursos de Educação Física, com titulação de mestrado e doutorado, das IES da Paraíba.

\begin{tabular}{|c|c|c|c|c|c|c|}
\hline IES/Titulação & UEPB & UFPB & UNIPÊ & FIP & FMN/JP & UNB \\
\hline $\begin{array}{c}\mathrm{N}^{\circ} \text { de Docentes } \\
M / D\end{array}$ & 29 & 36 & $\mathrm{NC}$ & 12 & 17 & 37 \\
\hline & & & & \multicolumn{2}{|c|}{ Total } & 131 \\
\hline
\end{tabular}

Dos 131 registros de produções catalogadas, registra-se que não foram disponibilizados os dados da UNIPÊ, percebe-se que a titulação de mestrado está em maior presença em todas as IES locais, mas mais expressiva nas privadas, e um pequeno número de doutores nas privadas em contraposição ao aumento de doutores nas públicas. Tal fato pode ser entendido por vários fatores: as universidades privadas são mais recentes; as universidades públicas têm maior investimento na formação dos professores; as 
universidades privadas optam por ter em seus quadros professores com titulação menor devido aos custos, dentre outros. As produções estão distribuídas nas IES conforme quadro 2.

Quadro 2 - Número de produções dos docentes dos Cursos de Educação Física, por titulação de mestrado e doutorado, das IES da Paraíba.

\begin{tabular}{|c|c|c|c|c|c|c|}
\hline IES/Titulação & UEPB & UFPB & UNIPE & FIP & FMN/JP & UNB \\
\hline Mestrado & 16 & 23 & $\mathrm{NC}$ & 10 & 15 & 19 \\
\hline Doutorado & 13 & 13 & $\mathrm{NC}$ & 02 & 02 & 18 \\
\hline & 29 & 36 & $\mathrm{NC}$ & 12 & 17 & 37 \\
\hline & & & & Total & \multicolumn{2}{|l|}{131} \\
\hline
\end{tabular}

No que se refere a origem das produções reconhecemos que há ainda uma grande parcela na região Sudeste, mas já conseguimos identificar um parcela expressiva na região Nordeste, conforme quadro 3.

Foi registrado um item identificado como Outras, devido a grande variedade de instituições de outras regiões, no entanto é importante anotar que a grande maioria é dos docentes da UNB que tem sua formação nesta mesma instituição. Importante destacar também a parcela de docentes que realizam suas pós-graduações em instituições estrangeiras, maior presença em Portugal.

Quadro 3 - Distribuição das produções dos docentes dos Cursos de Educação Física das IES da Paraíba por localização geográfica.

\begin{tabular}{|c|c|c|c|c|c|}
\hline Região & Nordeste & Sudeste & Outras & Exterior & Não Ident. \\
\hline Quantidade & 34 & 36 & 34 & 26 & 1 \\
\hline Percentual & $26 \%$ & $27,5 \%$ & $26 \%$ & 19,8 & $0,7 \%$ \\
\hline
\end{tabular}

Estes programas são majoritariamente na área de Educação Física, conforme distribuição do quadro 4. Na distribuição por ano de finalização, identificamos que a primeira foi no ano de 1992 (UNB), tendo uma concentração entre os anos 2003, 2004 e 2005 na UFPB; 2011 e 2012 na UEPB; 2003 e 2004 na UNB; 2012 na FMN/JP; e não há concentração na FIP, mas são de anos recentes.

$\mathrm{O}$ que nos permite reconhecer que boa parte dos docentes teve que recorrer a outras regiões pelo fato de que na região Nordeste o primeiro programa de pós-graduação em Educação Física na UPE/UFPB é recente, seguindo com a criação do programa da UFRN e da UFS. E mesmo indo a outras IES se inseriu em programas da área de Educação Física. Faz-se 
necessário reconhecer também a contribuição da área da Educação e das Ciências da Saúde nesta formação.

Quadro 4 - Distribuição das produções dos docentes dos Cursos de Educação Física das IES da Paraíba por programa de pós-graduação.

\begin{tabular}{|l|c|}
\hline \multicolumn{1}{|c|}{ Programa } & Quantidade \\
\hline Educação Física & 55 \\
\hline Ciências da Saúde & 19 \\
\hline Educação & 18 \\
\hline Ciências do Movimento Humano & 10 \\
\hline Saúde Coletiva & 05 \\
\hline Nutrição & 06 \\
\hline Medicina & 03 \\
\hline Filosofia & 02 \\
\hline Ciências Biomêdicas & 01 \\
\hline Teologia & 01 \\
\hline Exercise and health sciences & 01 \\
\hline Ciências do exercício & 01 \\
\hline Reabilitação & 01 \\
\hline Kinesiology & 01 \\
\hline Administração & 01 \\
\hline Saúde da Criança e do Adolescente & 01 \\
\hline Fisioterapia e Terapia Educacional & 01 \\
\hline Não Identificado & 04 \\
\hline
\end{tabular}

Identificamos também que das 131 produções, 67 receberam fomento, que na sua grande maioria foi oriunda da CAPES. Se retirarmos 23 fomentos da UNB, temos que nas IES localizadas na região apenas 44 receberam fomento para sua realização.

Fizemos uma primeira apreciação das áreas de estudos e observamos a concentração, em todas as IES, da produção na área de Atividade Física e Saúde, o que explicita a localização da nossa área no campo da saúde, mesmo que apareça de forma ainda discreta uma ampliação de áreas de inserção de nosso campo de conhecimento.

Teremos que refletir com maior profundidade, em uma nova etapa da pesquisa, nos objetos e problemáticas destas pesquisas, de forma a apontarmos como elas avançam ou não, frente às produções já identificadas em outras pesquisas, na reflexão da área de Educação Física no campo da Atividade Física e Saúde.

No que se refere aos grupos de pesquisa, observamos que os docentes da UFPB e UNB têm o maior número de grupos cadastrados e uma participação ampliada em grupos das próprias instituições e também 
participação em grupos de outras IES. Na FIP e FMN há participação de um grupo de professores em grupos de outras IES, visto a não existência de nenhum grupo da área nestas instituições. E na UEPB há dois grupos cadastrados, com participação também pequena dos docentes e apenas um caso de participação em grupo externo a instituição.

Importante registrar que a ocorrência de grupos de pesquisa é diretamente proporcional ao número de doutores de uma instituição, assim observamos uma ampliação dos grupos em instituições que tem esse número maior de doutores, mas também uma particularização das linhas que não permite ver um trabalho em diálogo entre os membros de um mesmo corpo docente.

As linhas de pesquisa apontam para uma atualização da discussão da área que vai muito além da produção dos docentes nas suas titulações, isso é muito importante, pois anuncia os avanços da área se refletindo na formação inicial, através especialmente das pesquisas.

Há outros dados que foram tratados estatisticamente e que mereceram nossa atenção de análise nesta etapa, mas que optamos por não incluí-los neste texto, para uma análise com maior profundidade em uma nova etapa.

\section{Considerações}

Ao reconhecer o cenário nacional de formação em Educação Física nossa pesquisa começa a demarcar sua intenção de compreender o percurso de formação da área, que busca entendê-la via a formação, no nível de pósgraduação stricto sensu, dos seus formadores.

O cenário do Estado da Paraíba, localizado nos dados iniciais, e agora ampliado, indica um crescimento necessário de cursos, mas que a rede pública (federal e estadual) não dá conta, desta forma as instituições privadas assumem este papel. Este fato é reconhecido em todas as áreas de formação, visto a ampliação do acesso ao ensino superior no Brasil, que se dá com diferentes políticas de governo, desde a ampliação das redes, a instituição de bolsas e programas de acesso aos alunos de baixa renda. Destacamos, no entanto, a necessidade de avaliação sistemáticas desses cursos. 
Percebe-se também que uma nova modalidade de ensino começa a ser inserida nesta região, e no caso da Paraíba é seu primeiro registro, que são os cursos à distância. Na Paraíba o curso instalado no formato a distância é de uma das mais importantes IES do Brasil, o que pode ser avaliado de forma positiva, mas também merece a atenção sistemática. É necessário destacar que estes cursos pretendem atingir municípios os mais diversos por todo o interior do estado, dando conta de uma demanda que as instituições locais não conseguem.

No que diz respeito à formação dos professores, os dados coletados indicam que cresce o nível de pós-graduação stricto sensu dos docentes dos cursos da Paraíba, podendo ser observado pelas duas instituições públicas. Na UFPB uma presença representativa já existente e na UEPB um crescimento significativo nos últimos anos, devido a uma formação interinstitucional, junto a UNESP, que também contribuiu com a formação de alguns docentes da UFPB. Em ambas, a contribuição da pós-graduação na região Sudeste é bem visível ainda.

Percebe-se que as IES estão buscando qualificar seu corpo docente, visto a necessidade de consolidar a tríade ensino-pesquisa-extensão, que se potencializará com a presença de novos doutores, que possibilitam a aquisição de financiamentos em nível nacional.

As duas faculdades privadas analisadas, no entanto, tem em seus quadros um número significativo de mestres, mas não de doutores, merecendo uma ampliação para que as mesmas possam assumir este mesmo papel no cenário paraibano, ou seja, garantir na formação inicial a tríade ensino-pesquisa-extensão.

Importante registrar, ainda, que foi registrada a presença de professores especialistas em todas as IES, mesmo que não sendo alvo de nosso estudo. Este dado precisa ser refletido por todas essas IES, visto a necessidade da qualificação dos docentes inseridos na formação universitária. Não estamos aqui querendo desqualificar o docente por sua titulação mínima, mas reconhecer a necessidade de ampliação da mesma, pois entendemos que esta qualificação reflete na formação da área.

Este cenário de crescimento indica a possibilidade de abertura de novos programas de pós-graduação stricto sensu. A UFPB já desenvolve um 
programa stricto sensu em parceria com a UPE, em nível de mestrado e sendo consolidado o doutorado este ano. Recentemente a UFRN e a UFS também criaram seus programas e estão em fase de consolidação de suas primeiras turmas.

Neste caso, a UEPB, sendo a outra instituição pública, é chamada a construir um projeto stricto sensu frente a seu novo quadro docente de doutores, de forma a termos mais uma possibilidade de formação dos docentes da área e ampliar a pesquisa acadêmica no estado.

Essa formação/produção dá sustentação à base da formação universitária, qual seja o ensino, a pesquisa e a extensão. Formação esta que vem merecendo inúmeros estudos que reconhecem o papel privilegiado da universidade para sua consolidação.

Desta forma, reconheço com Libâneo (1994) que o ensino, etapa integrante do processo de formação de professores, constitui o seu principal meio, não o único, mas o principal e que é a universidade seu palco de efetivação.

A pesquisa neste processo pode ser entendida, segundo Demo (2005), como um processo social que perpassa toda a vida acadêmica, do professor e do aluno. Ação sistemática que toma os problemas da formação como eixo e problematiza-os em seu cotidiano, dialogando com a realidade.

E a extensão é aqui entendida como uma situação educativa, situação esta em que professores e alunos assumem o papel de sujeitos cognoscentes, e eles são mediatizados pelo objeto que desejam conhecer (FREIRE, 1980).

Recorro à Constituição Federal do Brasil, nos termos do artigo 207 (BRASIL, 1988), onde afirma que "As universidades gozam de autonomia didático-científica, administrativa e de gestão financeira e patrimonial, e obedecerão ao princípio de indissociabilidade entre ensino, pesquisa e extensão". Indissociabilidade não questionada na atualidade, porém que apresenta dificuldade de ser efetivada nos projetos pedagógicos de diferentes áreas.

Para Moita e Andrade (2009, p. 273) a partir do princípio da indissociabilidade "[...] a extensão e a pesquisa tornam-se consequências naturais da docência, referências para que o ensino não se torne abstrato nem desligado das realidades locais". 
Reconheço, portanto, que o esforço de ampliar a formação dos docentes das IES vem no sentido de consolidar uma formação qualificada para esta universidade com formação ampla e socialmente referenciada.

Os dados que ora apresentamos já nos dá indícios de que a formação superior na Paraíba cresce e se qualifica, indicando por onde ela caminha frente às diferentes possibilidades desta área.

Conforme previsto, desde o projeto inicial, esta pesquisa entrará em uma nova etapa - III, onde vamos nos debruçar na identificação de por onde passa esta produção, reconhecendo para além de suas áreas, suas tendências teóricas e metodológicas, e seus indicadores na produção do conhecimento em Educação Física no Brasil.

\section{Referências}

ALBUQUERQUE. Joelma. A produção de pesquisa em Educação Física, esporte e lazer com a temática escola no nordeste brasileiro (1982-2004): possibilidades da educação para além do capital. 2007. Dissertação (Mestrado) - Mestrado em Educação, Faculdade de Educação, Universidade Federal da Bahia, Salvador, 2007.

BRACHT, Valter. Educaşão física \& ciência: cenas de um casamento (in)feliz. Ijuí: Unijuí, 1999.

BRASILEIRO, Lívia Tenorio; SILVA, Roseane. Produção científica em Educação Física no nordeste do Brasil: analisando a produção dos docentes nas instituições formadoras do estado da Paraíba - Etapa I - Relatório Final de Iniciação Científica - PIBIC 2010/2011. Campina Grande: UEPB, 2011.

CHAVES, Márcia; SÁNCHEZ GAMBOA, Silvio; TAFFAREL, Celi. (Org.). Prática pedagógica e produção do conhecimento na educação física \& esporte e lazer. Maceió: Ed UFAL, 2003.

CHAVES, Márcia. A produção do conhecimento em Educação Física nos Estados do Nordeste (Alagoas, Babia, Pernambuco e Sergipe) 1982-2004: balanço e perspectivas. 2005. Tese (Pós-doutorado) - Pós-Graduação em Educação, Faculdade de Educação, Universidade Federal da Bahia, Salvador, 2005.

CHAVES-GAMBOA, Márcia; SÁNCHEZ GAMBOA, Silvio. Pesquisa na Educação Física: epistemologias, escola e formação profissional. Maceió: Ed UFAL, 2009.

DEMO, Pedro. Metodologia científica em Ciências Sociais. São Paulo: Atlas, 1981. DEMO, Pedro. Pesquisa: princípio científico e educativo. São Paulo: Cortez, 2005. 
FARIA JÚNIOR, Alfredo Gomes. Pesquisa em educação física: enfoques e paradigmas. In: FARIA JÚNIOR, A. G. de; FARINATTI, P. de T.V. (Org.). Pesquisa e producão do conhecimento em Educação Física: livro do ano de 1991. Rio de Janeiro: Ao Livro Técnico, 1991. p. 13-33.

FIP. Site Oficial. Disponível em: http://www.fiponline.com.br. Acesso: 12 de abril de

2012.

FMN. Site Oficial. Disponível em: http://mauriciodenassau.edu.br/. Acesso: 12 de abril de 2012.

FREIRE, Paulo. Extensão ou comunicaşão? 5. ed. Rio de Janeiro: Paz e Terra, 1980.

LIBÂNEO, Jose Carlos. Didática. São Paulo: Cortez, 1994.

MOITA, Filomena Maria; ANDRADE, Fernando Cézar. Ensino-pesquisaextensão: um exercício de indissociabilidade na pós-graduação. Revista Brasileira Educação. Rio de Janeiro, v.14, n.41, 2009, p. 269-280.

LÖWY, Michel. Ideologia e Ciências Sociais. São Paulo: Cortez, 1986.

SÁNCHEZ GAMBOA. Silvio. Análise epistemológica dos métodos na pesquisa educacional. 1982. Dissertação (Mestrado) - Pós-Graduação em Educação, Faculdade de Educação, Universidade de Brasília, Brasília, 1982.

SÁNCHEZ GAMBOA. Silvio. Epistemologia da pesquisa em educação: estruturas lógicas e tendências metodológicas. 1987. Tese (Doutorado) - Pós-Graduação em Educação, Faculdade de Educação, Universidade Estadual de Campinas, Campinas, 1987.

SÁNCHEZ GAMBOA. Silvio. Epistemologia da Educaşão Física: as inter-relações necessárias. 1. ed. Maceió: Ed UFAL, 2007.

SILVA, Rossana Valéria. Mestrados em Educação Física no Brasil: pesquisando a suas pesquisas. 1990. Dissertação (Mestrado) - Pós-Graduação em Educação Física, Universidade de Santa Maria, Santa Maria, 1990.

UEPB. Site Oficial. Disponível em: <www.uepb.edu.br>. Acesso em: 12 de abril de 2012.

UFPB. Site Oficial. Disponível em: <www.ufpb.br>. Acesso em: 12 de abril de 2012.

UNB. Site Oficial. Disponível em: http://www.unb.br/. Acesso: 12 de abril de 2012.

UNIPE. Site Oficial. Disponível em: <www.unipe.br>. Acesso em: 12 de abril de 2012 . 